\title{
VENTILATOR ASSOCIATED PNEUMONIA - CLINICAL PROFILE, COMORBIDITIES AND PROGNOSIS
}

Sreenivasa Rao Sudulagunta1, L. Sreenivasa Murthy22, Shiva Kumar Banglore Raja3 , Mahesh Babu Sodalagunta 4 , Mona Sepehrar5, Munawar Dhanish Mohammed 6 , Sony Parethu Sunny ${ }^{7}$, Rajdeepak V. S ${ }^{8}$

${ }^{1}$ Senior Resident, Department of Internal Medicine, Dr. B. R. Ambedkar Medical College, KG Halli, Bangalore.

2 Professor, Department of Medicine, Dr. B. R. Ambedkar Medical College, KG Halli, Bangalore.

${ }^{3}$ Professor, Department of Medicine, Dr. B. R. Ambedkar Medical College, KG Halli, Bangalore.

${ }^{4}$ Postgraduate, Department of General Medicine, K. S. Hegde Medical College.

${ }^{5}$ Doctor of Pharmacy, Baptist Hospital, Bangalore.

${ }^{6}$ Postgraduate, Department of General Medicine, Dr. B. R. Ambedkar Medical College, KG Halli, Bangalore.

7 Postgraduate, Department of General Medicine, Dr. B. R. Ambedkar Medical College, KG Halli, Bangalore.

${ }^{8}$ Postgraduate, Department of General Medicine, Dr. B. R. Ambedkar Medical College, KG Halli, Bangalore.

ABSTRACT

\section{BACKGROUND}

Ventilator-associated pneumonia occurs in 48-72 hours following endotracheal intubation, characterised by the presence of a new infiltrate and signs of systemic infection. This retrospective study was done to evaluate clinical profile, epidemiological, laboratory findings and outcome of VAP for the evaluation of management strategies.

\section{MATERIALS AND METHODS}

This retrospective study analysed data of all adult patients diagnosed/admitted with VAP between January 2008 and January 2016. The medical records were analysed for the demographic data (Age, sex), clinical features, comorbid conditions, investigations, mode and results of the treatment and complications of the procedures.

\section{RESULTS}

A total of 189 VAP episodes were observed during the study period. A total of 30 patients were excluded due to inadequate data, second episode of VAP and other causes. The mean incidence rate of VAP overall was 6.97 per 1000 ventilator-days. The mean age was $41.7 \pm 19.8$ years (range 16 -80 years) and among all the patients, $68.55 \%$ patients $(121 / 159)$ were less than the age of 60 years. The mean Sequential Organ Failure Assessment (SOFA) score (range 1-15) on admission was highest among admissions to medical ICU $[\mathrm{P}=0.0034]$. Single organism was cultured from respiratory samples of $73 / 159$ (46\%) patients and $\geq 2$ organisms isolated from another 77/159 (48.4\%) patients and cultures were negative in 9 patients. The most common bacteria isolated was Pseudomonas followed by Acinetobacter, Klebsiella, Haemophilus and Enterobacter. The 30-day mortality in VAP study was observed to be $47 / 159$ $(29.55 \%)$.

\section{CONCLUSIONS}

The VAP incidence has progressively decreased over the years due to preventive measures and better management. Patients admitted with neurological disorder are at increased risk of death. Smoking, bedbound status, head trauma, respiratory failure, cardiovascular disease, duration of mechanical ventilation and inadequate antibiotic therapy significantly increase the mortality risk in VAP patients. High resistance to antibiotics may be due to excessive and uncontrolled usage and the pattern of resistance should be used to devise a plan to stop improper use.

\section{KEYWORDS}

VAP, Mechanical Ventilation, ARDS, SOFA Score.

HOW TO CITE THIS ARTICLE: Sudulagunta SR, Murthy LS, Raja SKB, et al. Ventilator associated pneumonia- Clinical profile, comorbidities and prognosis. J. Evolution Med. Dent. Sci. 2016;5(94):6953-6958, DOI: 10.14260/jemds/2016/1573

\section{BACKGROUND}

Ventilator-associated pneumonia (VAP) is defined as pneumonia that occurs in 48-72 hours or thereafter following endotracheal intubation, characterised by the presence of a new or progressive infiltrate, signs of systemic infection

Financial or Other, Competing Interest: None.

Submission 03-11-2016, Peer Review 15-11-2016,

Acceptance 18-11-2016, Published 24-11-2016.

Corresponding Author:

Dr. Sreenivasa Rao Sudulagunta,

Senior Resident,

Department of Internal Medicine,

Dr. B. R. Ambedkar Medical College,

KG Halli, Bangalore-560045.

E-mail: drssreenivasarao@gmail.com,dr.sreenivas@live.in DOI: $10.14260 /$ jemds/2016/1573

\section{(c) (i) $\$$}

(fever, altered white blood cell count), changes in sputum characteristics, and detection of a causative agent.[1] Ventilator-associated pneumonia (VAP) occurs due to the microorganism invasion of the lower respiratory tract including lung parenchyma. Intubation leads to compromise in the integrity of the oropharynx and trachea and predisposes the oral and gastric secretions entry in to the lower airways.

VAP contributes to about $50 \%$ of all cases of hospitalacquired pneumonia.[1],[2] Estimates of VAP varies from 9 to 27 $\%$ of all mechanically ventilated patients, and the risk is found to be highest during the early course of hospitalisation. ${ }^{[1],[3]}$ VAP is observed to be the second most common nosocomial infection in the intensive care unit and the most common infection in mechanically ventilated patients. [4],[5] VAP ranges from 1.2 to 8.5 per 1,000 ventilator days.[6] Risk is highest during the initial 5 days of starting of mechanical ventilation 
(3\%) with the mean duration between intubation and VAP development being 3.3 days. ${ }^{[7]}$

This risk reduces to $2 \%$ per day from day 5 to day 10 of mechanical ventilation, and $1 \%$ per day thereafter.[1],[8] Earlier studies reported the attributable mortality for VAP from 33 to 50\%.[1] Several studies have noted different results of attributable mortality, because of different populations (Lessacute trauma, acute respiratory distress syndrome [ARDS], and medical and surgical ICU) and variation in empirical therapy during the first 2 days. The organisms causing infection have an important impact on outcome, with greater mortality rates due to Pseudomonas aeruginosa, Acinetobacter spp., and Stenotrophomonas maltophilia.

VAP incidence varies in different studies depending on the diagnostic criteria, ICU type, and patients. The causative organisms also vary based on patient demographics, the duration of hospital stay, and antibiotic policy used. Incidence and the associated organisms needs to be studied according to local conditions for effective management of VAP. Our retrospective study aims to describe the clinical profile, causative organisms, and outcome of VAP in ICU patients.

\section{MATERIALS AND METHODS}

The retrospective study analysed data of all adult patients diagnosed/admitted with VAP between January 2008 and January 2016. Data was pooled from 3 hospitals with facilities of medical or surgical intensive care units. The medical records were analysed for the demographic data (Age, sex), clinical features, comorbid conditions, investigations, mode and results of the treatment and complications of the procedures. Centre of Disease Control (CDC) defined VAP as a pneumonia that occurs in a patient who has been intubated and ventilated for 2 or more calendar days on the date of the event.

Pneumonia was defined as the presence of new or progressive pulmonary infiltrates or consolidation or cavitation in chest radiography, associated with at least 2 of the following criteria: Body temperature of $>38^{\circ} \mathrm{C}$ or $<36^{\circ} \mathrm{C}$ with no other known cause, leucocytes count $<4000 / \mathrm{mm}^{3}$ or $>12000 / \mathrm{mm}^{3}$, and purulent tracheal secretion or a change in characteristics of an existing secretion.[9] Sequential Organ Failure Assessment (SOFA) score was used for clinical severity assessment.[10],[11]

The multidrug resistant pathogens were: Extendedspectrum beta-lactamase producing Gram-negative Enterobacteriaceae, Pseudomonas aeruginosa, methicillinresistant Staph. aureus, and non-fermenting pathogens (Stenotrophomonas maltophilia, Acinetobacter baumannii,) were resistant to 3 or more antibiotic classes described: Antipseudomonal penicillins or cephalosporins, aminoglycosides. fluoroquinolones, and carbapenems.[12] If the empirical therapy was started before 48 hours after VAP diagnosis with inclusion of at least one antimicrobial agent to which the organism was susceptible in the antibiogram, then it is considered as appropriate.[13]

Gram stain, culture-sensitivity, either tracheal aspirate or bronchoalveolar lavage and other necessary investigations were done in all included patients. SPSS (Statistical Package for Social Sciences, V18) was used for statistical analysis. A p value of $<0.05$ is considered as statistically significant.

\section{RESULTS}

A total of 189 VAP episodes were observed during the study period. A total of 30 patients were excluded due to inadequate data, second episode of VAP and other causes. The remaining 159 patients with complete records were thoroughly analysed. The mean incidence rate of VAP overall was 6.97 per 1000 ventilator-days. The ventilator associated pneumonia rates were 8.37 per 1000 ventilator-days in 2008, 7.58 per 1000 ventilator-days in 2009, 7.24 per 1000 ventilator-days in 2010, 6.38 per 1000 ventilator-days in 2011, 6.68 per 1000 ventilator-days in 2012, 6.18 per 1000 ventilator-days in 2013, 6.17 per 1000 ventilator-days in 2014, and 4.88 per 1000 ventilator-days in 2015.

The comparison between the mean VAP rates in MICU and SICU during the study period were represented in figure 1 . The mean age was $41.7 \pm 19.8$ years (Range $16-80$ years) and among all the patients, $68.55 \%$ patients $(121 / 159)$ were less than the age of 60 years [Figure 2]. Males constituted $83.01 \%$ $(132 / 159)$ and females constituted $16.98 \%$ (27/159) of all patients [Figure 3]. Regarding age distribution, 71 patients (44.65\%) were in 41-50 years age group, 29 patients (18.23\%) were in 16-40 years age group, 20 patients $(12.57 \%)$ were in 71-80 years age group and 18 patients $(11.32 \%)$ were in 6170 years age group. The comorbidities associated with VAP in study group are represented in table 1 . The commonest comorbidity in the VAP study group was hypertension $(103 / 159,64.77 \%)$, followed by diabetes mellitus $(93 / 159$, $58.49 \%)$, cardiovascular disease $(37 / 159,23.27 \%)$, respiratory disease $(37 / 159,23.27 \%)$, neurological disorder (23/159, 14.46\%), liver disease $(20 / 159,12.57 \%)$, followed by thyroid and renal disease $(13 / 159,8.18 \%)$ and malignancy (7/159, 4.40\%).

The admission diagnoses of VAP study are represented in table 2. The commonest admission diagnoses were polytrauma $(47 / 159,29.55 \%)$, sepsis $(39 / 159,24.52 \%)$, cardiovascular disease $(23 / 159,17.61 \%)$, respiratory failure (27/159, 16.98\%), head trauma (21/159, 13.20\%), abdominal disease $(20 / 159,12.57 \%)$, and neurological disease $(13 / 159$, 8.17\%). The mean Sequential Organ Failure Assessment (SOFA) score (range 1-15) on admission was highest among admissions to medical ICU [P=0.0034]. SOFA score, functional status, and smoking status are represented at table 3 . Mechanical ventilation duration was longest in patients admitted to medical ICU before the VAP diagnosis (Range 248 days).

Inadequate antibiotic therapy was observed in 35 (22.01\%) patients. Smoking history was observed in 35 $(22.01 \%)$ patients. Bedbound status was observed in 22 (13.83\%) patients. Endotracheal tube aspiration was done in $156 / 159$ (98.11\%) patients and bronchoalveolar lavage was done in the remaining $37 / 159$ (23.27\%) patients. Single organism was cultured from respiratory samples of 73/159 (46\%) patients and $\geq 2$ organisms isolated from another $77 / 159$ (48.4\%) patients and cultures were negative in 9 patients. The antibiotic sensitivity pattern of the commonest microorganisms isolated were represented in table 4 .

Enterobacteriaceae were found to be resistant to 15 antibiotics and Klebsiella was found to have resistance to 16 antibiotics. The most common bacteria isolated was Pseudomonas followed by Acinetobacter, Klebsiella, Haemophilus and Enterobacter. The 30-day mortality in VAP study was observed to be $47 / 159$ (29.55\%). Statistically significant differences between discharged patients and expired patients were observed in the age of patients $(p<0.001)$, number of comorbidities $(p<0.001)$, diabetes 
mellitus $(\mathrm{p}=0.001)$, hypertension $(\mathrm{p}=0.01)$, cardiovascular disease $(p=0.001)$, neurological disorder $(p=0.03)$, respiratory disease $(p=0.003)$, admission diagnoses of head trauma $(p=0.03)$, polytrauma $(p=0.05)$, respiratory failure $(p=0.001)$, neurological disease $(p=0.04)$, cardiovascular disease $(\mathrm{p}=0.03)$, abdominal disease $(\mathrm{p}=0.01)$, SOFA score on admission $(p=0.007)$, duration of mechanical ventilation before VAP onset $(p=0.01)$, late or early onset of VAP $(p=0.001)$, and inadequate antibiotic therapy $(p=0.001)$. There was no statistically significant association between specific pathological organism and mortality.

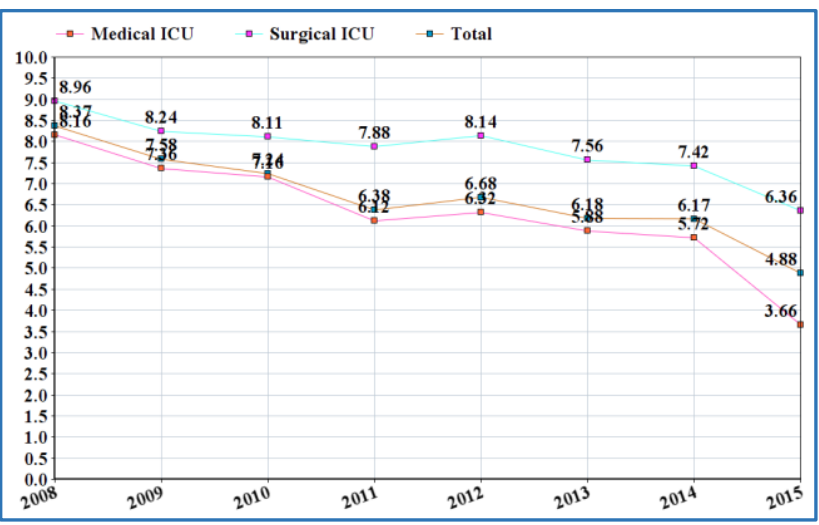

Figure 1. Comparison between the Mean VAP Rates in MICU and SICU during the Study Period

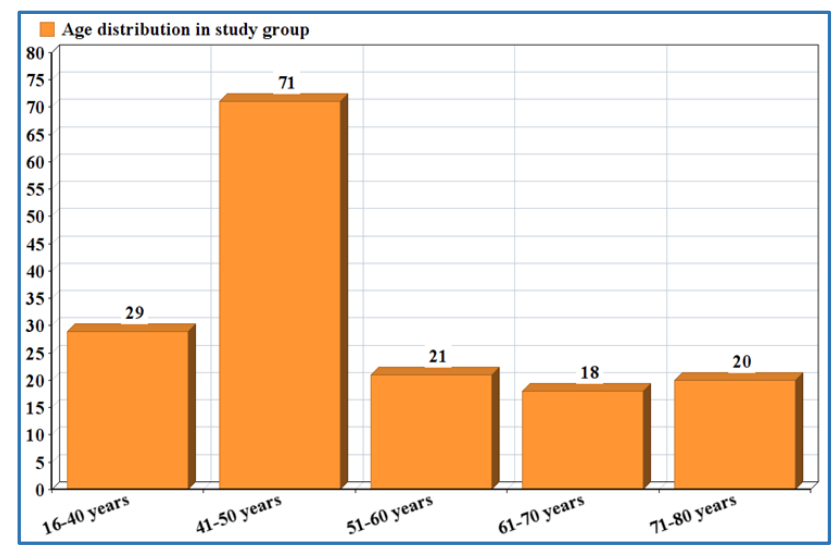

Figure 2. Age Distribution of Study Group

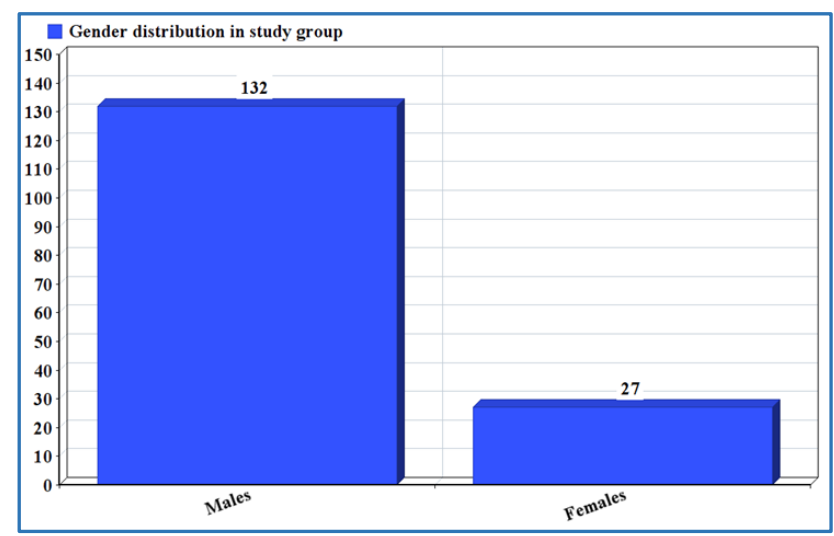

Figure 3: Sex Distribution in Study Group

\begin{tabular}{|c|c|c|c|}
\hline Comorbidities & $\begin{array}{c}\text { Medical } \\
\text { ICU(91) }\end{array}$ & $\begin{array}{c}\text { Surgical } \\
\text { ICU(68) }\end{array}$ & Total(159) \\
\hline Diabetes & 76 & 17 & 93 \\
Mellitus & $(83.51 \%)$ & $(25 \%)$ & $(58.49 \%)$ \\
\hline Hypertension & 82 & 21 & 103 \\
$(90.10 \%)$ & $(30.88 \%)$ & $(64.77 \%)$ \\
\hline $\begin{array}{c}\text { Cardiovascular } \\
\text { Disease }\end{array}$ & $\begin{array}{c}25 \\
(27.47 \%)\end{array}$ & $\begin{array}{c}12 \\
(17.64 \%)\end{array}$ & $\begin{array}{c}37 \\
(23.27 \%)\end{array}$ \\
\hline Thyroid & 9 & 4 & 13 \\
Disorder & $(9.89 \%)$ & $(5.88 \%)$ & $(8.18 \%)$ \\
\hline Renal Disease & 9 & 4 & 13 \\
& $(9.89 \%)$ & $(5.88 \%)$ & $(8.18 \%)$ \\
\hline Neurological & 17 & 6 & 23 \\
Disorder & $(18.68 \%)$ & $(8.82 \%)$ & $(14.46 \%)$ \\
\hline Respiratory & 26 & 11 & 37 \\
Disease & $(28.57 \%)$ & $(16.17 \%)$ & $(23.27 \%)$ \\
\hline Liver Disease & $\begin{array}{c}15 \\
(16.48 \%)\end{array}$ & $\begin{array}{c}5 \\
(7.35 \%)\end{array}$ & $\begin{array}{c}20 \\
(12.57 \%)\end{array}$ \\
\hline Malignancy & 4 & 3 & 7 \\
& $(4.39 \%)$ & $(4.41 \%)$ & $(4.40 \%)$ \\
\hline \multicolumn{2}{|c|}{ Table 1. Comorbidities in VAP Study Group } \\
\hline \multicolumn{4}{|c|}{}
\end{tabular}

\begin{tabular}{|c|c|c|c|}
\hline $\begin{array}{c}\text { Admission } \\
\text { Diagnosis }\end{array}$ & $\begin{array}{l}\text { Medical } \\
\text { ICU(91) }\end{array}$ & $\begin{array}{l}\text { Surgical } \\
\text { ICU(68) }\end{array}$ & $\begin{array}{l}\text { Total } \\
\text { (159) }\end{array}$ \\
\hline Head Trauma & $\begin{array}{c}4 \\
(4.39 \%)\end{array}$ & $\begin{array}{c}17 \\
(25 \%)\end{array}$ & $\begin{array}{c}21 \\
(13.20 \%)\end{array}$ \\
\hline Polytrauma & $\begin{array}{c}2 \\
(2.20 \%)\end{array}$ & $\begin{array}{c}45 \\
(66.17 \%)\end{array}$ & $\begin{array}{c}47 \\
(29.55 \%)\end{array}$ \\
\hline $\begin{array}{l}\text { Respiratory } \\
\text { Failure }\end{array}$ & $\begin{array}{c}22 \\
(24.17 \%)\end{array}$ & $\begin{array}{c}5 \\
(7.35 \%)\end{array}$ & $\begin{array}{c}27 \\
(16.98 \%)\end{array}$ \\
\hline Sepsis & $\begin{array}{c}28 \\
(30.76 \%)\end{array}$ & $\begin{array}{c}11 \\
(16.17 \%)\end{array}$ & $\begin{array}{c}39 \\
(24.52 \%) \\
\end{array}$ \\
\hline $\begin{array}{c}\text { Neurological } \\
\text { Disease }\end{array}$ & $\begin{array}{c}11 \\
(12.08 \%)\end{array}$ & $\begin{array}{c}2 \\
(2.94 \%)\end{array}$ & $\begin{array}{c}13 \\
(8.17 \%)\end{array}$ \\
\hline $\begin{array}{l}\text { Cardiovascular } \\
\text { Disease }\end{array}$ & $\begin{array}{c}16 \\
(17.58 \%)\end{array}$ & $\begin{array}{c}7 \\
(10.29 \%)\end{array}$ & $\begin{array}{c}23 \\
(17.61 \%)\end{array}$ \\
\hline $\begin{array}{l}\text { Abdominal } \\
\text { Disease }\end{array}$ & $\begin{array}{c}3 \\
(3.29 \%)\end{array}$ & $\begin{array}{c}17 \\
(25 \%)\end{array}$ & $\begin{array}{c}20 \\
(12.57 \%)\end{array}$ \\
\hline Miscellaneous & $\begin{array}{c}3 \\
(3.29 \%)\end{array}$ & $\begin{array}{c}4 \\
(5.88 \%)\end{array}$ & $\begin{array}{c}7 \\
(4.40 \%)\end{array}$ \\
\hline
\end{tabular}

\begin{tabular}{|c|c|c|c|}
\hline Variables & $\begin{array}{l}\text { Medical } \\
\text { ICU (91) }\end{array}$ & $\begin{array}{c}\text { Surgical ICU } \\
(68)\end{array}$ & $\begin{array}{l}\text { Total } \\
(159)\end{array}$ \\
\hline $\begin{array}{l}\text { SOFA Score } \\
(\text { Mean } \pm \text { SD) }\end{array}$ & $8.1 \pm 4.1$ & $6.3 \pm 3.3$ & $7.6 \pm 3.6$ \\
\hline $\begin{array}{c}\text { Duration of } \\
\text { Mechanical } \\
\text { Ventilation before } \\
\text { VAP Onset } \\
\text { (Mean } \pm \text { SD) } \\
\text { (in Days) }\end{array}$ & $18.4 \pm 12.8$ & $13.6 \pm 8.9$ & $14.7 \pm 9.1$ \\
\hline $\begin{array}{c}\text { Inadequate } \\
\text { Antibiotic } \\
\text { Therapy }\end{array}$ & $\begin{array}{c}24 \\
(26.37 \%)\end{array}$ & $11(16.17 \%)$ & $\begin{array}{c}35 \\
(22.01 \%)\end{array}$ \\
\hline Smoker & $\begin{array}{c}21 \\
(23.07 \%)\end{array}$ & $14(20.58 \%)$ & $\begin{array}{c}35 \\
(22.01 \%)\end{array}$ \\
\hline $\begin{array}{l}\text { Ambulatory } \\
\text { Status }\end{array}$ & $\begin{array}{c}86 \\
(94.50 \%)\end{array}$ & $51(75 \%)$ & $\begin{array}{c}137 \\
(86.16 \%)\end{array}$ \\
\hline $\begin{array}{l}\text { Bedbound } \\
\text { Status }\end{array}$ & $\begin{array}{c}5 \\
(5.49 \%)\end{array}$ & $\begin{array}{c}17 \\
(25 \%)\end{array}$ & $\begin{array}{c}22 \\
(13.83 \%)\end{array}$ \\
\hline \multicolumn{4}{|c|}{$\begin{array}{l}\text { Table 3. SOFA Scores, Smoking } \\
\text { Status and Functional Status }\end{array}$} \\
\hline
\end{tabular}




\begin{tabular}{|c|c|c|c|c|c|c|c|c|c|}
\hline 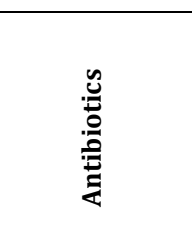 & 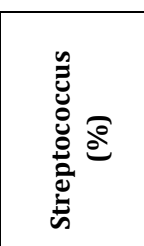 & 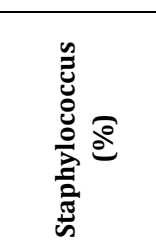 & $\underbrace{\circ}_{\text {도 }}$ & 氖 & 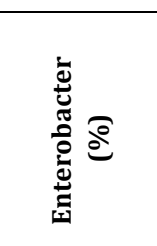 & 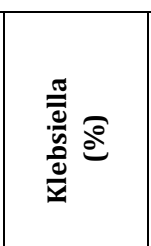 & 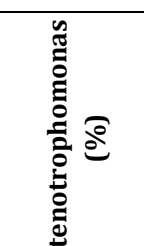 & 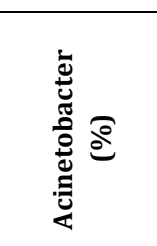 & 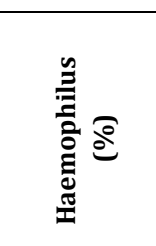 \\
\hline Penicillin-G & $3(33.3 \%)$ & $12(38.7 \%)$ & - & 19 (34.5\%) & - & - & - & - & $11(33.3 \%)$ \\
\hline Oxacillin & - & $21(67.7 \%)$ & - & - & - & - & - & - & - \\
\hline Ampicillin & - & $11(35.5 \%)$ & $4(40 \%)$ & $21(38.2 \%)$ & $11(35.5 \%)$ & 7 (17.1\%) & - & $9(21.4 \%)$ & $30(90.9 \%)$ \\
\hline $\begin{array}{c}\text { Amoxicillin } \\
\text { /Clavulanate }\end{array}$ & $9(100 \%)$ & - & $5(50 \%)$ & $28(50.9 \%)$ & $15(48.4 \%)$ & $10(24.4 \%)$ & - & $5(11.9 \%)$ & $31(93.9 \%)$ \\
\hline $\begin{array}{c}\text { Piperacillin } \\
\text { /Tazobactam }\end{array}$ & - & $5(16.1 \%)$ & $6(60 \%)$ & $52(94.5 \%)$ & $26(83.9 \%)$ & $35(85.4 \%)$ & - & $12(28.6 \%)$ & - \\
\hline Cefuroxime & $4(44.4 \%)$ & - & $3(30 \%)$ & - & $15(48.4 \%)$ & $21(51.2 \%)$ & - & - & $27(81.8 \%)$ \\
\hline Ceftriaxone & $9(100 \%)$ & $4(12.9 \%)$ & $3(30 \%)$ & - & $15(48.4 \%)$ & $21(51.2 \%)$ & - & $17(40.5 \%)$ & - \\
\hline Ceftazidime & - & - & $4(40 \%)$ & $47(85.5 \%)$ & $15(48.4 \%)$ & \begin{tabular}{|c|}
- \\
\end{tabular} & - & $16(38.1 \%)$ & $25(75.8 \%)$ \\
\hline Cefepime & - & - & $3(30 \%)$ & $43(78.2 \%)$ & - & $22(53.7 \%)$ & - & $17(40.5 \%)$ & - \\
\hline Clindamycin & $7(77.8 \%)$ & $28(90.3 \%)$ & - & - & $19(61.3 \%)$ & $16(39 \%)$ & - & - & $14(42.4 \%)$ \\
\hline Erythromycin & $5(55.6 \%)$ & $24(77.4 \%)$ & - & - & $4(12.9 \%)$ & $5(12.2 \%)$ & - & - & - \\
\hline Vancomycin & - & $31(100 \%)$ & - & - & $30(96.8 \%)$ & $4(9.75 \%)$ & - & - & - \\
\hline Cotrimoxazole & - & - & $9(90 \%)$ & - & $31(100 \%)$ & $29(70.7 \%)$ & $8(100 \%)$ & $29(69 \%)$ & $28(84.8 \%)$ \\
\hline Ciprofloxacin & - & - & $5(50 \%)$ & $55(100 \%)$ & $31(100 \%)$ & $35(85.4 \%)$ & - & $31(73.8 \%)$ & - \\
\hline Levofloxacin & - & $29(93.5 \%)$ & - & $21(38.2 \%)$ & $30(96.8 \%)$ & $33(80.5 \%)$ & 7 (87.5\%) & - & - \\
\hline Gentamicin & - & $28(90.3 \%)$ & $6(60 \%)$ & $45(81.8 \%)$ & $31(100 \%)$ & $30(73.2 \%)$ & - & $17(40.5 \%)$ & - \\
\hline Meropenem & $2(22.2 \%)$ & $2(6.45 \%)$ & \begin{tabular}{|c|}
10 \\
$(100 \%)$
\end{tabular} & 27 (49.1\%) & $31(100 \%)$ & $39(95.1 \%)$ & - & $33(78.6 \%)$ & $12(36.4 \%)$ \\
\hline Colistin & - & - & $2(20 \%)$ & $55(100 \%)$ & - & 15 (36.6\%) & - & $41(97.6 \%)$ & - \\
\hline Ertapenem & $1(11.1 \%)$ & $5(16.1 \%)$ & $9(90 \%)$ & $25(45.5 \%)$ & $31(100 \%)$ & $31(75.6 \%)$ & - & $39(92.9 \%)$ & $12(36.4 \%)$ \\
\hline $\begin{array}{c}\text { Total Number } \\
\text { of Isolates }\end{array}$ & $\begin{array}{c}9 \\
(100 \%)\end{array}$ & $\begin{array}{c}31 \\
(100 \%)\end{array}$ & \begin{tabular}{|c|}
10 \\
$(100 \%)$
\end{tabular} & $\begin{array}{c}55 \\
(100 \%)\end{array}$ & $\begin{array}{c}31 \\
(100 \%)\end{array}$ & \begin{tabular}{|c|}
41 \\
$(100 \%)$
\end{tabular} & $\begin{array}{c}8 \\
(100 \%)\end{array}$ & $\begin{array}{c}42 \\
(100 \%)\end{array}$ & $\begin{array}{c}33 \\
(100 \%)\end{array}$ \\
\hline
\end{tabular}

\begin{tabular}{|c|c|c|c|}
\hline Variables & $\begin{array}{c}\text { Discharged } \\
\text { (112) }\end{array}$ & $\begin{array}{c}\text { Expired } \\
(47)\end{array}$ & P value \\
\hline \multicolumn{4}{|l|}{ Age } \\
\hline Mean \pm SD (in years) & $44.3 \pm 18.6$ & $59.9 \pm 15.9$ & $<0.0001$ \\
\hline$\leq 60$ years $(121)$ & $100(89.3 \%)$ & $21(44.7 \%)$ & \multirow{2}{*}{$<0.001$} \\
\hline$>60$ years $(38)$ & $12(10.7 \%)$ & $26(55.3 \%)$ & \\
\hline \multicolumn{4}{|l|}{ Gender } \\
\hline Males (132) & $92(82.1 \%)$ & $40(85.1 \%)$ & \multirow{2}{*}{0.65} \\
\hline Females (27) & $20(17.9 \%)$ & $7(14.9 \%)$ & \\
\hline \multicolumn{4}{|l|}{$\begin{array}{c}\text { Number of } \\
\text { Comorbidities }\end{array}$} \\
\hline 0 & $71(63.4 \%)$ & $3(6.38 \%)$ & $<0.001$ \\
\hline 1 & $31(27.7 \%)$ & $15(31.9 \%)$ & 0.589 \\
\hline$\geq 2$ & $10(8.9 \%)$ & $29(61.7 \%)$ & $<0.001$ \\
\hline Comorbidities & & & \\
\hline Diabetes Mellitus (93) & $53(57 \%)$ & $40(43 \%)$ & \multirow[b]{2}{*}{0.001} \\
\hline $\begin{array}{c}\text { No diabetes } \\
\text { Mellitus (66) }\end{array}$ & $59(89.4 \%)$ & 7 (10.6\%) & \\
\hline Hypertension (103) & $66(64 \%)$ & $37(36 \%)$ & \multirow[b]{2}{*}{0.01} \\
\hline $\begin{array}{c}\text { No } \\
\text { Hypertension (56) }\end{array}$ & $46(82.1 \%)$ & $10(17.9 \%)$ & \\
\hline $\begin{array}{l}\text { Cardiovascular } \\
\text { Disease (37) }\end{array}$ & $14(37.8 \%)$ & $23(62.2 \%)$ & \multirow[b]{2}{*}{0.001} \\
\hline $\begin{array}{c}\text { Without } \\
\text { Cardiovascular } \\
\text { Disease (122) }\end{array}$ & $98(80.3 \%)$ & $24(19.7 \%)$ & \\
\hline $\begin{array}{c}\text { Renal } \\
\text { Disease (13) }\end{array}$ & $7(53.8 \%)$ & $6(46.2 \%)$ & \multirow{2}{*}{0.17} \\
\hline $\begin{array}{c}\text { No Renal } \\
\text { Disease (146) }\end{array}$ & $105(71.9 \%)$ & $41(28.1 \%)$ & \\
\hline $\begin{array}{l}\text { Neurological } \\
\text { Disorder (23) }\end{array}$ & $12(52.2 \%)$ & $11(47.8 \%)$ & \multirow{2}{*}{0.03} \\
\hline $\begin{array}{l}\text { No Neurological } \\
\text { Condition (136) }\end{array}$ & $100(73.5 \%)$ & $36(26.5 \%)$ & \\
\hline
\end{tabular}

\begin{tabular}{|c|c|c|c|}
\hline $\begin{array}{c}\text { Respiratory Disease } \\
\text { (37) }\end{array}$ & $19(51.4 \%)$ & $18(48.6 \%)$ & \multirow{2}{*}{0.003} \\
\hline $\begin{array}{l}\text { No Respiratory } \\
\text { Disease (122) }\end{array}$ & $93(76.2 \%)$ & $29(23.8 \%)$ & \\
\hline Liver Disease (20) & $11(55 \%)$ & $9(45 \%)$ & \multirow[b]{2}{*}{0.10} \\
\hline $\begin{array}{c}\text { No Liver } \\
\text { Disease (139) }\end{array}$ & $101(72.7 \%)$ & $38(27.3 \%)$ & \\
\hline Malignancy (7) & $5(71.4 \%)$ & $2(28.6 \%)$ & \multirow{2}{*}{0.95} \\
\hline No Malignancy (152) & $107(70.4 \%)$ & $45(29.6 \%)$ & \\
\hline \multicolumn{4}{|l|}{ Associated Factors } \\
\hline Smoker (35) & $21(60 \%)$ & $14(40 \%)$ & \multirow{2}{*}{0.12} \\
\hline Non-smoker (124) & $91(73.4 \%)$ & $33(26.6 \%)$ & \\
\hline \multicolumn{4}{|l|}{ Functional Status } \\
\hline Ambulatory (137) & $100(73 \%)$ & $37(27 \%)$ & \multirow{2}{*}{0.07} \\
\hline Bedbound (22) & $12(54.5 \%)$ & $10(45.5 \%)$ & \\
\hline \multicolumn{4}{|l|}{$\begin{array}{c}\text { Admission } \\
\text { Diagnosis }\end{array}$} \\
\hline Head Trauma (21) & $19(90.5 \%)$ & $2(9.5 \%)$ & 0.03 \\
\hline Polytrauma (47) & $28(59.6 \%)$ & $19(40.4 \%)$ & 0.05 \\
\hline $\begin{array}{l}\text { Respiratory } \\
\text { Failure (27) }\end{array}$ & $7(25.9 \%)$ & $20(74.1 \%)$ & 0.001 \\
\hline Sepsis (39) & $24(61.5 \%)$ & $15(38.5 \%)$ & 0.16 \\
\hline $\begin{array}{l}\text { Neurological } \\
\text { Disease (13) }\end{array}$ & $6(46.1 \%)$ & $7(53.9 \%)$ & \multirow{2}{*}{$\begin{array}{l}0.04 \\
0.03\end{array}$} \\
\hline $\begin{array}{c}\text { Cardiovascular } \\
\text { Disease (23) }\end{array}$ & $12(52.2 \%)$ & $11(47.8 \%)$ & \\
\hline $\begin{array}{c}\text { Abdominal } \\
\text { Disease (20) }\end{array}$ & $19(95 \%)$ & $1(5 \%)$ & 0.01 \\
\hline SOFA Score on & & & \\
\hline $\begin{array}{l}\text { Admission } \\
(\text { Mean } \pm \text { SD })\end{array}$ & $6.4 \pm 3.7$ & $8.1 \pm 3.3$ & 0.007 \\
\hline Duration of & $13.7 \pm 8.4$ & $17.1 \pm 7.8$ & 0.01 \\
\hline
\end{tabular}




\begin{tabular}{|c|c|c|c|}
\hline $\begin{array}{c}\text { Mechanical } \\
\text { Ventilation Before } \\
\text { VAP } \\
\text { Onset (in days) } \\
\text { (Mean } \pm \text { SD) }\end{array}$ & & & \\
\hline Onset of VAP & & & \\
\hline Early ( $\leq 4$ days) (83) & $76(91.6 \%)$ & $7(8.4 \%)$ & 0.001 \\
\hline Late ( $>4$ days) (76) & $36(47.4 \%)$ & $40(52.6 \%)$ & 0.001 \\
\hline $\begin{array}{c}\text { Inappropriate } \\
\text { Antibiotic } \\
\text { Therapy (35) }\end{array}$ & $15(42.9 \%)$ & $20(57.1 \%)$ & 0.001 \\
\hline \multicolumn{4}{|c|}{$\begin{array}{l}\text { Table 5. Association of Variables and } \\
\text { Outcome of Patients in VAP Study }\end{array}$} \\
\hline
\end{tabular}

\section{DISCUSSION}

Our study reports an overall VAP incidence of 6.97 per 1000 ventilator-days. The VAP incidence has progressively decreased over the years due to preventive measures and better management. The complex interplay of the presence of endotracheal tube, risk factors, virulence of the organisms and immunity of the patient result in the VAP development. The presence of an endotracheal tube is the important risk factor which results in damage of natural defence mechanisms i.e., cough reflex of glottis and larynx, which prevents microaspiration around the cuff of the endotracheal tube.[14] Virulent bacteria can enter the lower respiratory tract by the following mechanisms: (1) Microaspiration. (2) Biofilm development laden with bacteria in the endotracheal tube (commonly Gram-negative bacteria and fungal species). (3) Secretion pooling and trickling around the endotracheal tube cuff. (4) Mucociliary secretion clearance impairment.[15],[16]

Virulent organisms can colonise the stomach, sinuses, nasopharynx and oropharynx, replacing the normal flora.[16],[17] The positive pressure exerted by the ventilator gives constant thrust to the virulent organism enriched material. The use of NIV (Non-invasive positive pressure ventilation) has been found to be associated with low VAP rates compared to invasive ventilation and re-intubation following extubation dramatically increases the VAP rates. Host factors that increase risk for VAP include the underlying diseases, previous surgeries and antibiotic usage. Critically ill patients have impaired phagocytosis and are functionally immunosuppressed even before the onset of nosocomial infection.[18],[19] This dysfunction is due to the anaphylatoxin C5a, which impairs phagocytic activity of neutrophils. [18]

Newer studies show that a combined T-cell, monocyte, and neutrophil dysfunction has been useful to predict risk of nosocomial infection.[19] Elevation of regulatory T-cells, monocyte deactivation (HLA-DR expression) and neutrophil dysfunction (CD88 expression) have shown usefulness in infection prediction in the critically ill compared to healthy controls.[19] Centres for Disease Control and Prevention (CDC) recommended a new surveillance criteria for possible or probable VAP.[20] The goals were to find frequent ventilator complications, improvement of surveillance for public reporting by allowing comparability across health centres.[21] As per these criteria, at least 2 days of stable or decreasing ventilator settings (minimum positive end-expiratory pressure [PEEP] or fraction of inspired oxygen [ $\mathrm{FiO}_{2}$ ] everyday) followed by higher settings for at least 2 more days is required for the patient to be diagnosed with a ventilatorassociated condition (VAC).

Most ventilator-associated conditions i.e., pulmonary oedema, atelectasis, pneumonia, and ARDS, have researched prevention and management strategies.[22] Presence of purulent secretions and positive culture will label as possible or probable VAP. Patients with a VAC and purulent secretions or positive cultures alone have "possible pneumonia"; and those with both purulent secretions and positive cultures have "probable pneumonia". Chest radiograph findings have been excluded, because of their subjectivity. However, presently the CDC algorithm is only for surveillance purposes.

Our study supports the findings of a multicentre study from Greece which reported $45 \%$ of VAP cases due to trauma (polytrauma 29\% and head injury 16\%).[23] Increased VAP incidence has been observed in patients with neurological disorders.[24] In our study, $14.46 \%$ (23/159) of the VAP patients were admitted due to neurological disease and 30-day mortality in these patients was $47.8 \%(11 / 23)$. Our study shows that the incidence of VAP decreased over the years due to awareness and better management strategies. Male patients are at more risk for development of VAP (83\% vs. 17\%). Our study supports the already observed fact that females are at less risk, but mortality is higher i.e. $37 \% .^{[25]}$

The mortality rate in our study was about $30 \%$, which is similar to other studies.[26] Our study also supports the observation that elderly population are at more risk of death due to VAP i.e. 55\% in our study[27] [Table 5]. Comorbidities have significant effect in the onset of VAP and mortality. [13] The increased number of comorbidities pose a greater risk of mortality in VAP patients as observed in our study, patients with $\geq 2$ comorbidities have been observed to have mortality of $61.7 \%(p<0.01)$ [Table 5]. Inadequate or half-hearted antibiotic therapy was observed to be a major risk factor for increasing mortality in our study $(57 \%)(\mathrm{p}<0.01)$. Our study supports other studies that reported similar risk due to inadequate therapy.[28],[29]

\section{CONCLUSION}

We put forward the following observations based on our retrospective analysis. This retrospective study has the limitations in sample size and accurate calculation of incidence. Male patients have more incidence of VAP and females have higher mortality. Elderly patients are at more risk of VAP and have higher mortality rate. Patients admitted to medical ICU have more comorbidities and increased number of comorbidities increases the risk of mortality. Patients admitted with neurological disorder have increased risk of death. The mean SOFA scores are higher in patients admitted in medical ICU. Smoking, bedbound status, head trauma, respiratory failure, cardiovascular disease, duration of mechanical ventilation and inadequate antibiotic therapy significantly increase the mortality risk in VAP patients.

Our study found high resistance to Piperacillin /tazobactam, Meropenem, Ertapenem and levofloxacin. This resistance may be due to excessive and uncontrolled usage of higher antibiotics for the diseases that doesn't warrant their use. The antibiotic sensitivity pattern should be compared with other studies and a plan must be devised to stop improper use of antibiotics leading to resistance and complications. 


\section{REFERENCES}

1. American Thoracic Society, Infectious Diseases Society of America. Guidelines for the management of adults with hospital-acquired, ventilator-associated, and healthcareassociated pneumonia. Am J Respir Crit Care Med 2005;171(4):388-416.

2. Vincent JL, Bihari DJ, Suter PM, et al. The prevalence of nosocomial infection in intensive care units in Europe. Results of the European prevalence of infection in intensive care (EPIC) study. EPIC international advisory committee. JAMA 1995;274(8):639-44.

3. Chastre J, Fagon JY. State of the art: ventilator-associated pneumonia. Am J Respir Crit Care Med 2002;165(7):867903.

4. Hunter JD. Ventilator associated pneumonia. BMJ 2012, 344: e3325. 10.1136/bmj.e3325

5. Afshari A, Pagani L, Harbarth S. Year in review 2011: Critical care - infection. Crit Care 2012;16:242-7.

6. Skrupky LP, McConnell K, Dallas J, et al. A comparison of ventilator-associated pneumonia rates as identified according to the National Healthcare Safety Network and American College of Chest Physicians Criteria. Crit Care Med 2012;40(1):281-4.

7. Rello J, Ollendorf DA, Oster G, et al. Epidemiology and outcomes of ventilator-associated pneumonia in a large US database. Chest 2000;122(6):2115-21.

8. Cook DJ, Walter SD, Cook RJ, et al. Incidence of and risk factors for ventilator-associated pneumonia in critically ill patients. Ann Int Med 1998;129(6):433-40.

9. Johanson WG, Pierce AK, Sanford JP, et al. Nosocomial respiratory infections with gram-negative bacilli. The significance of colonization of the respiratory tract. Annals of Internal Medicine 1972;77(5):701-6.

10. Torres A, Carlet J. Ventilator-associated pneumonia. European task force on ventilator-associated pneumonia. European Respiratory Journal 2001;17(5):1034-45.

11. Vincent JL, de Mendonça A, Cantraine F, et al. Use of the SOFA score to assess the incidence of organ dysfunction/failure in intensive care units: results of a multicentre, prospective study. Working group on "sepsis-related problems" of the European Society of Intensive Care Medicine. Critical Care Medicine 1998;26(11)1793-1800.

12. Depuydt PO, Vandijck DM, Bekaert MA, et al. Determinants and impact of multidrug antibiotic resistance in pathogens causing ventilator-associatedpneumonia. Critical Care 2008;12(6):R142.

13. Resende MM, Monteiro SG, Callegari B, et al. Epidemiology and outcomes of ventilator-associated pneumonia in northern Brazil: an analytical descriptive prospective cohort study. BMC Infectious Diseases 2013;13:119.

14. Zolfaghari PS, Wyncoll DL. The tracheal tube: gateway to ventilator-associated pneumonia. Crit Care 2011;15(5): 310.

15. Grgurich PE, Hudcova J, Lei Y, et al. Diagnosis of ventilator-associated pneumonia: controversies and working toward a gold standard. Curr Opin Infect Dis 2013;26(2):140-50.
16. Mietto C, Pinciroli R, Patel N, et al. Ventilator associated pneumonia: evolving definitions and preventive strategies. Respir Care 2013;58(6):990-1007.

17. Rocha LA, Ribas MR, da Darini CAL, et al. Relationship between nasal colonization and ventilator-associated pneumonia and the role of the environment in transmission of Staphylococcus aureus in intensive care units. Am J Infect Control 2013;41(12):1236-40.

18. Morris AC, Brittan M, Wilkinson TS, et al. C5a-mediated neutrophil dysfunction is RhoA-dependent and predicts infection in critically ill patients. Blood 2011;117(19): 5178-88.

19. Morris CA, Anderson N, Brittan M, et al. Combined dysfunctions of immune cells predict nosocomial infection in critically ill patients. $\mathrm{Br} \mathrm{J}$ Anaesth 2013;111(5):778-87.

20. National Healthcare Safety Network (NHSN). CDC/NHSN Protocol Clarifications 2013.

[http://www.cdc.gov/nhsn/PDFs/pscManual/10VAE_FI NAL.pdf]

21. Klompas M. Complications of mechanical ventilation - the CDC's new surveillance paradigm. N Engl J Med 2013; 368(16):1472-5.

22. Hayashi Y, Morisawa K, Klompas M, et al. Toward improved surveillance: the impact of ventilatorassociated complications on length of stay and antibiotic use in patients in intensive care units. Clin Infect Dis 2013;56(4):471-7.

23. Apostolopoulou E, Bakakos P, Katostaras T, et al. Incidence and risk factors for ventilator-associated pneumonia in 4 multidisciplinary intensive care units in Athens, Greece. Respiratory Care 2003;48(7):681-8.

24. Mehndiratta MM, Nayak R, Ali S, et al. Ventilators in ICU: a boon or burden. Annals of Indian Academy of Neurology 2016;19(1):69-73.

25. Sharpe JP, Magnotti LJ, Weinberg JA, et al. Gender disparity in ventilator-associated pneumonia following trauma: identifying risk factors for mortality. Journal of Trauma and Acute Care Surgery 2014;77(1):161-5.

26. Alp E, Voss A. Ventilator associated pneumonia and infection control. Annals of Clinical Microbiology and Antimicrobials 2006;5:7.

27. Blot S, Koulenti D, Dimopoulos G, et al. Prevalence, risk factors and mortality for ventilator-associated pneumonia in middle-aged, old and very old critically ill patients. Critical Care Medicine 2014;42(3):601-9.

28. Inchai J, Pothirat $\mathrm{C}$, Liwsrisakun $\mathrm{C}$, et al. Ventilatorassociated pneumonia: epidemiology and prognostic indicators of 30-day mortality. Japanese Journal of Infectious Diseases 2015;68(3):181-6.

29. Luna CM, Vujacich $P$, Niederman MS, et al. Impact of BAL data on the therapy and outcome of ventilator-associated pneumonia. Chest 1997;111(3):676-85. 